\title{
LUMINESCÊNCIA E ABSORÇÃO ÓPTICA DE NANOFIBRAS DOS ÓXIDOS DE TITÂNIO E TUNGSTÊNIO NA REGIÃO DO UV-VISÍVEL
}

Luminescence and optical absorption of titanium and tungsten oxides nanofibers in the uv-visible region

\section{Luana Góes Soares da Silva ${ }^{1}$ Annelise Kopp Alves ${ }^{2}$}

RESUMO: A síntese de fibras por electrospinning nos proporciona a obtenção de nanoestruturas unidimensionais com boa flexibilidade, propriedades ópticas, catalíticas, elevada área superficial e porosidade. Podem ser utilizadas em aplicações como: catálises, células solares, células combustíveis, membranas, baterias de hidrogênio, entre outras. Sendo assim, neste trabalho sintetizamos nanofibras de $\mathrm{TiO}_{2}$ e de $\mathrm{TiO}_{2} / \mathrm{WO}_{3}$ por electrospinning. Estas nanofibras foram tratadas termicamente entre $650^{\circ} \mathrm{C}$ e $800^{\circ} \mathrm{C}$, e caracterizadas quanto: a morfologia por microscopia eletrônica de varredura $(\mathrm{MEV})$, fases cristalinas por difração de raios $\mathrm{X}(\mathrm{DRX})$, fotoatividade mediante ensaios de degradação de $125 \mathrm{~mL}$ de uma solução $20 \mathrm{ppm}$ do corante alaranjado de metila e por colorimetria. Os resultados parciais, apontam a amostra de $\mathrm{TiO}_{2} / \mathrm{WO}_{3}$ tratada termicamente à $800{ }^{\circ} \mathrm{C}$ mais efetiva na degradação do corante e na reflexão das diferentes cores, possivelmente devido à existência de uma sincronicidade entre as propriedades químicas e físicas dos óxidos de titânio e tungstênio.

Palavras-chave: Luminescência. Nanofibras. Titânio, Tungstênio.

ABSTRACT: The synthesis of fibers by electrospinning allows us to obtain one-dimensional nanostructures with good flexibility, optical, catalytic properties, high surface area and porosity. They can be used in applications such as: catalysis, solar cells, fuel cells, membranes, hydrogen batteries, among others. Therefore, in this work we synthesized $\mathrm{TiO}_{2}$ and $\mathrm{TiO}_{2} / \mathrm{WO}_{3}$ nanofibers by electrospinning. These nanofibers were heat treated between $650^{\circ} \mathrm{C}$ and $800{ }^{\circ} \mathrm{C}$, and characterized as: the morphology by scanning electron microscopy (SEM), crystalline phases by X-ray diffraction (XRD), photoactivity through tests of degradation of $125 \mathrm{~mL}$ of a solution $20 \mathrm{ppm}$ of orange methyl dye and by colorimetry. The partial results indicate the $\mathrm{TiO}_{2} / \mathrm{WO}_{3}$ sample heat treated at $800{ }^{\circ} \mathrm{C}$ more effective in the degradation of the dye and in the reflection of the different colors, possibly due to the existence of a synchronicity between the chemical and physical properties of the titanium and tungsten oxides.

Keywords: Luminescence, Nanofibers, Titanium, Tungsten.

\section{INTRODUÇÃO}

Propriedades ópticas compreendem a resposta de um material quando submetido à radiação eletromagnética e, em particular a luz visível [r]. O comportamento óptico de um material sólido é o resultado de suas interações, com a radiação eletromagnética e comprimentos de onda dentro da

\footnotetext{
1 Pesquisadora, Dra a em Engenharia - área de Concentração: Ciência e Tecnologia dos Materiais da Universidade Federal do Rio Grande do Sul. E-mail: lugoes.soares@gmail.com.

${ }^{2}$ Doutora em Engenharia na área de concentração Ciência e Tecnologia dos Materiais pela Universidade Federal do Rio Grande do Sul. E-mail: annelise.alves@ufrgs.br.
} 
região visível do espectro [I]. A capacidade de percepção das ondas eletromagnéticas pelos olhos humanos é compreendida em comprimentos de onda $(\lambda)$ entre 380 e $780 \mathrm{~nm}$. A rigor, o olho humano capta três diferentes estímulos de cor: azul, verde e vermelho. A união destes três estímulos define as reproduções coloridas no cérebro, dessa maneira qualquer cor pode ser constituída pela adição de vermelho, verde e azul $[2,3,4]$.

Quando a luz incide sobre a superfície de um objeto, podem ocorrer um ou mais fenômenos correspondentes à cor: transmissão, absorção ou reflexão [5]. A luminescência em materiais sólidos diz respeito ao processo de emissão de luz. Pode ocorrer através da fotoluminescência e a eletroluminescência. Determina a quantidade de luz que é percebida em determinada cor, ou seja, a capacidade de ser clara ou escura [6].

A cor é determinada pela disposição espectral de radiação emitida, transmitida ou refletida. A composição da luz é observada através do efeito da cor no objeto, por comprimentos de onda e pela maneira que o objeto altera a luz que irradia sobre ele. Essas alterações podem ser demonstradas por curvas de absorbância, transmitância ou de reflectância espectral [I]. É uma informação visual, gerada por um estímulo que é percebido pelo nosso olho e interpretado pelo nosso cérebro. Para que os olhos sejam capazes de perceber uma cor é fundamental presença de raios de luz. Os raios de luz refletidos nos olhos percorrem um longo trajeto até chegar na retina. Lá se localizam numa região central da retina chamada fóvea. O olho humano possui dois tipos de células que são responsáveis por nos fazer enxergar: os cones e os bastonetes. Os bastonetes são células que necessitam de pouca luz para serem sensibilizadas. Entretanto não conseguem formar imagens coloridas ou nítidas. Já os cones são sensibilizados com uma grande quantidade de luz e geram imagens nítidas e coloridas [7].

Existem 3 tipos de cones: os azuis, os vermelhos, e os verdes. O cone azul é ativado em comprimentos de onda curtos, o cone verde é ativado em comprimentos de onda médio e o cone vermelho é ativado em comprimentos de onda longos. Assim, as cores supracitadas são os três tipos de coloração que os nossos olhos captam. Todas as outras cores que vemos são formadas a partir destas 3 cores [7].

A percepção visual colorida é particular e no espectro visível depende da iluminação. Sendo assim, neste trabalho avaliamos a luminescência e como os olhos percebem a região de absorbância de cores por nanofibras de $\mathrm{TiO}_{2}$ e de $\mathrm{TiO}_{2} / \mathrm{WO}_{3}$ quando irradiadas com luz visível.

\section{MATERIAIS E MÉTODOS Electrospinning}

As nanofibras foram obtidas através do preparo de 2 soluções precursoras. A solução de $\mathrm{TiO}_{2}$ foi obtida através da mistura de 2,5 $\mathrm{mL}$ de propóxido de titânio (TIP), 2,o $\mathrm{mL}$ de ácido acético glacial 
e 5,0 mL de uma solução alcoólica contendo I0\% em peso de polivinilpirrolidona (PVP). A solução de $\mathrm{TiO}_{2} / \mathrm{WO}_{3}$ foi preparada misturando os reagentes acima mencionados acrescida de $\mathrm{I} \mathrm{mL}$ de peróxido de hidrogênio e o,ıo $\mathrm{g}$ de $\mathrm{H}_{2} \mathrm{WO}_{4}$, que foram mantidos sob agitação magnética por is minutos. Em seguida, uma seringa de plástico foi carregada com $5 \mathrm{~mL}$ da solução precursora de $\mathrm{TiO}_{2}$ ou de $\mathrm{TiO}_{2} / \mathrm{WO}_{3}$ que foi conectada a uma agulha de alimentação de aço inoxidável hipodérmica por uma fonte de alta tensão. A distância entre o tubo capilar e o coletor cilíndrico foi de $12 \mathrm{~cm}$, a tensão foi de $13,5 \mathrm{kV}$ com um fluxo de $\mathrm{I}, 8 \mathrm{~mL} / \mathrm{h}$. O coletor cilíndrico foi recoberto com uma folha de alumínio para coletar as nanofibras produzidas a cada 30 minutos por um período de 4 horas. As nanofibras foram tratadas termicamente em um forno elétrico (SANCHIS) a $650{ }^{\circ} \mathrm{C}, 700{ }^{\circ} \mathrm{C}, 750^{\circ}{ }^{\circ} \mathrm{C}$ ou $800 \stackrel{\circ}{\circ}$ com patamar de $\mathrm{I}$ h e taxa de aquecimento de $\mathrm{I}, 4 \mathrm{C} / \mathrm{min}$.

\section{Caracterização}

Utilizou-se um difratômetro PHILIPS com radiação $\mathrm{CuK} \alpha$, com tensão de $40 \mathrm{kV}$ e $40 \mathrm{~mA}$, equipado com o software X'PERT HightScore, para identificar as fases presentes nas fibras. Um microscópio eletrônico de varredura (MEV, JEOL 6o6o) equipado com EDS (espectroscopia de energia dispersiva), usado para avaliar a morfologia das nanofibras e identificar a presença de átomos de $\mathrm{W}$, Ti e $\mathrm{O}$ nas amostras, dependendo da composição das nanofibras. O equipamento utilizado para medir a energia de band gap foi um espectrofotômetro de feixe duplo UV-vis- NIR (Cary 50oo), com uma esfera integradora no modo de reflexão difusa de luz. Os valores da energia de band gap foram obtidos através da correlação de Kubelka e Munk. A colorimetria foi determinada por um espectofotômetro (Konica-Minolta, CM 2600 d) equipado com um software. O equipamento possui uma esfera integrada a um filtro ultra-violeta. $\mathrm{O}$ iluminante utilizado foi D65, que corresponde a faixa espectral da luz do dia, e o observador utilizado foi o que corresponde a um campo de visão de ${ } 0^{\circ}$. O equipamento registra as informações para definir o índice de cor com base no sistema Cie$\mathrm{La}^{*} \mathrm{~b}$. Neste sistema, a cor é determinada conforme os valores positivos ou negativos das coordenadas $\mathrm{a}^{*}$ (vermelho e verde) e $\mathrm{b}^{*}$ (amarelo e azul). $\mathrm{O} \mathrm{L}^{*}$ define a luminescência variando entre o a $100 \%$.

O desempenho fotocatalítico das nanofibras de $\mathrm{TiO}_{2}$ e de $\mathrm{TiO}_{2} / \mathrm{WO}_{3}$ foi analisado através da alteração na concentração do corante alaranjado de metila, sob irradiação UVA. O processo de fotocatálise foi realizado em um reator fotocatalítico, de vidro pyrex, onde a radiação foi proporcionada por I2 lâmpadas negras UVA, de $8 \mathrm{~W}$ cada, modelo Flúor BLB T5 e da marca Sadokin. As lâmpadas estão dispostas em dois semi-cilindros, que possuem superfície interna refletora. Os outros componentes do reator fotocatalítico incluem; um agitador magnético, um sistema de aeração de ar comprimido e um banho termostático. Para a execução dos ensaios fotocatalíticos, as nanofibras 
de $\mathrm{TiO}_{2}$ ou de $\mathrm{TiO}_{2} / \mathrm{WO}_{3}$ foram misturadas a $125 \mathrm{~mL}$ de uma solução contendo 20 ppm do corante alaranjado de metila. A mistura foi colocada em um ultrassom (Cole-Parmer CP-750), por is minutos. A solução foi então transferida para o reator fotocatalítico, sob constante agitação, e a temperatura de $30^{\circ} \mathrm{C}$. Fez-se borbulhar ar no período de exposição à luz UVA. Antes do início de cada ensaio, uma amostra de $4 \mathrm{~mL}$ desta solução foi coletada e definida como amostra inicial. Durante o ensaio, com sistema de luz UVA ligado, foram retiradas com uma seringa de plástico, alíquotas de $4 \mathrm{~mL}$ da solução, em intervalos de 15 minutos, filtradas em filtros de $0,2 \mu \mathrm{m}$ e, colocadas em cubetas de polimetilmetacrilato (PMMA) para em seguida serem analisadas por espectrofotometria.

\section{RESULTADOS E DISCUSSÃO}

A Figura I apresenta o difratograma das nanofibras sintetizadas por electrospinning. As amostras antes do tratamento térmico apresentaram-se amorfas para todas as formulações. As nanofibras de $\mathrm{TiO}_{2}$ (Figura Ia) tratadas até a temperatura de $700{ }^{\circ} \mathrm{C}$ apresentaram somente a presença da fase cristalina anatase (JCPDS o10782486), com o primeiro pico característico em aproximadamente $2 \Theta=25,27 \mathrm{I}^{\circ}$. As nanofibras tratadas a partir de $750^{\circ} \mathrm{C}$ apresentaram além da fase anatase, a fase rutilo (JCPDS oI-077-0442), com o primeiro pico característico em aproximadamente $2 \Theta=27,294^{\circ}$, resultado da ocorrência de uma transição de fases do $\mathrm{TiO}_{2}$, previsto após o aumento da temperatura de tratamento térmico [8,9]. Nas nanofibras de $\mathrm{TiO}_{2} / \mathrm{WO}_{3}$ (Figura $\mathrm{Ib}$ ) tratadas até $650{ }^{\circ} \mathrm{C}$ foram identificadas as fases anatase (JCPDS or-078-2486) e brookita (JCPDS or-075-1582) para $\mathrm{TiO}_{2}$ com picos característicos em aproximadamente $2 \theta=25,27 \mathrm{I}^{\circ}$ e $25,425^{\circ}$, respectivamente. As nanofibras tratadas a partir de $700{ }^{\circ} \mathrm{C}$ apresentaram para o $\mathrm{TiO}_{2}$ as fases anatase, brookita e rutilo (JCPDS or-077-0442), com o primeiro pico característico em aproximadamente $2 \Theta=27,294^{\circ}$. Para $\mathrm{WO}_{3}$ a fase monoclínica (JCPDS oo-032-1393) apareceu em todas as temperaturas de tratamento térmico, com o primeiro pico característico a aproximadamente $2 \theta=23^{\circ}$.

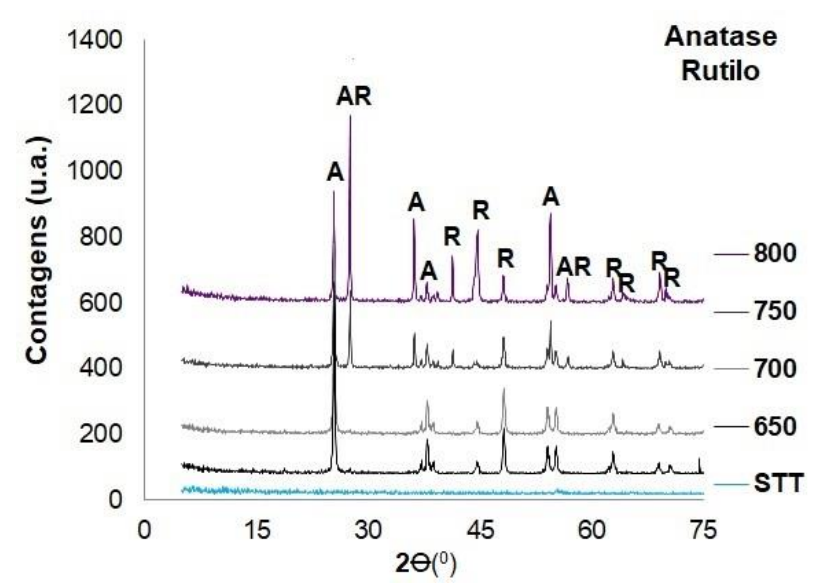

(a)

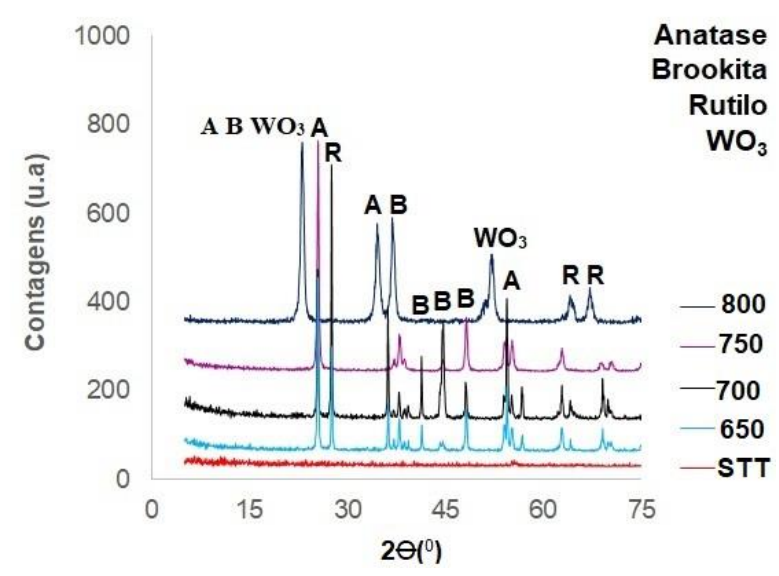

(b) 
Figura I. Difratograma das nanofibras de (a) $\mathrm{TiO}_{2}$ e de (b) $\mathrm{TiO}_{2} / \mathrm{WO}_{3}$.

A Figura $2(\mathrm{a}-\mathrm{b})$ apresenta as imagens de microscopia eletrônica de varredura (MEV) da superfície das nanofibras de $\mathrm{TiO}_{2}$ e de $\mathrm{TiO}_{2} / \mathrm{WO}_{3}$, tratadas termicamente a $800 \stackrel{\circ}{\circ}$, respectivamente. Pelas imagens, nota-se que as nanofibras de $\mathrm{TiO}_{2}$ e de $\mathrm{TiO}_{2} / \mathrm{WO}_{3}$ aparentam morfologia similar, parecem ser constituídas de um aglomerado de partículas primárias, com forma alongada, contínua e sem uma orientação preferencial.

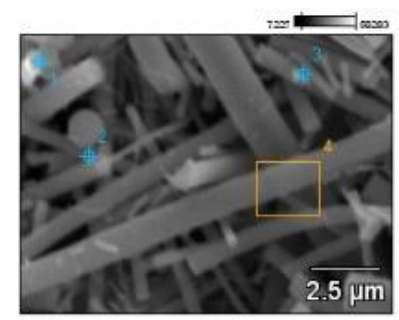

Full scale counts: 1024

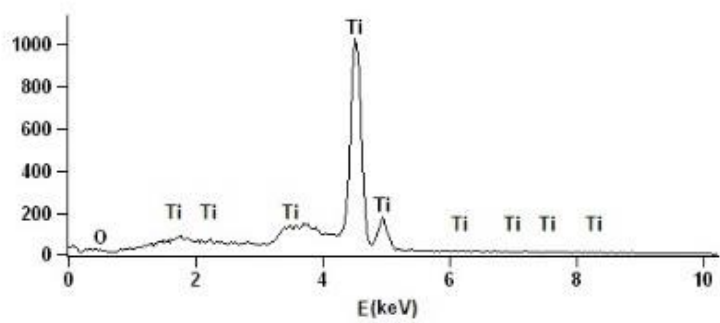

(a)

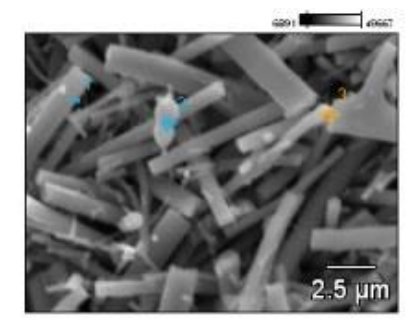

Full scale counts: 1000

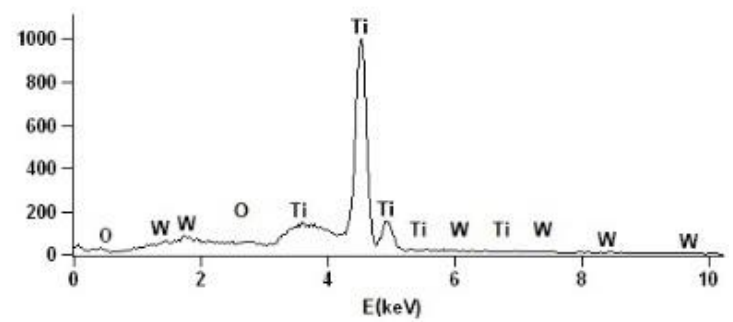

(b)

Figura 2. Imagens de microscopia eletrônica de varredura (MEV) das nanofibras de (a) $\mathrm{TiO}_{2}$ e de (b) $\mathrm{TiO}_{2} / \mathrm{WO}_{3}$ tratadas a $800 \stackrel{\circ}{-} \mathrm{C}$.

A Figura $3(\mathrm{a}-\mathrm{b})$ apresenta a atividade fotocatalítica das nanofibras de $\mathrm{TiO}_{2}$ e de $\mathrm{TiO}_{2} / \mathrm{WO}_{3}$ na degradação do corante alaranjado de metila durante 135 minutos de exposição à luz UVA $(\lambda=365$ $\mathrm{nm})$. Todas as nanofibras apresentaram atividade fotocatalítica. Para as nanofibras de $\mathrm{TiO}_{2}$, as mais fotoativas foram as que receberam tratamento térmico a $650{ }^{\circ} \mathrm{C}$, conseguiram degradar aproximadamente $40 \%$ do corante alaranjado de metila em 135 minutos de irradiação UVA. As nanofibras tratadas a $700{ }^{\circ} \mathrm{C}$ e o padrão $\mathrm{P}_{25}$ tiveram um comportamento similar, degradaram aproximadamente $30 \%$ do corante em 135 minutos de irradiação UVA. E as nanofibras tratadas a 750 ${ }^{\circ} \mathrm{C}$ e $800{ }^{\circ} \mathrm{C}$, degradaram aproximadamente $20 \%$ e $10 \%$, respectivamente, do corante em 135 minutos de irradiação UVA.

Este decréscimo observado na fotoatividade das amostras é o resultado da formação da fase rutilo, que no caso das nanofibras surge a partir de tratamentos acima de $700{ }^{\circ} \mathrm{C}$. A forma rutilo é 
menos fotoativa do que a forma anatase e, por esta razão o seu aparecimento reduz a atividade fotocatalítica das nanofibras sintetizadas $[8,9]$.

A presença de tungstênio nas amostras de $\mathrm{TiO}_{2} / \mathrm{WO}_{3}$ aumentou a atividade fotocatalítica das nanofibras tratadas a temperatura de $700{ }^{\circ} \mathrm{C}$, $750{ }^{\circ} \mathrm{C}$ e $800{ }^{\circ} \mathrm{C}$, para aproximadamente $36 \%$, $50 \%$ e 90\% de degradação, respectivamente. Tal efetividade se deve; a redução do band gap de 3,05 eV até 2,89 eV , inibição da recombinação do par elétron/lacuna $\left[(e) /\left(h^{+}\right)\right]$, que permitiu a transferência de cargas entre o $\mathrm{TiO}_{2}$ e o $\mathrm{WO}_{3}$, e ao aumento na formação de defeitos pontuais (vacâncias de $\mathrm{O}_{2}$ ). A elevação da temperatura de tratamento térmico possibilitou que as vacâncias de $\mathrm{O}_{2}$ adquirissem a mobilidade necessária para passar para um estado desordenado na rede, aumentando a capacidade de degradação e a eficiência do processo [10,II,I2].

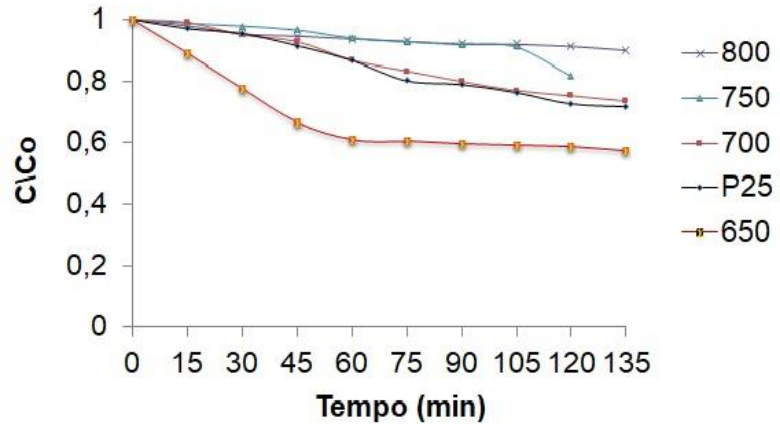

(a)

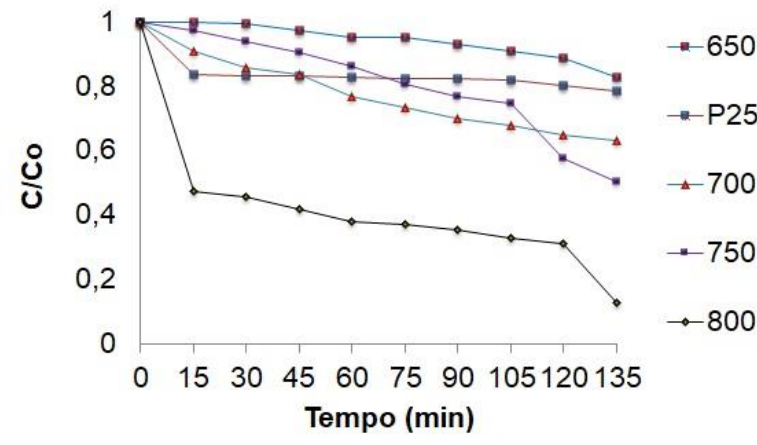

(b)

Figura 3. Atividade fotocatalítica das nanofibras na degradação da concentração relativa do corante alaranjado de metila: (a) $\mathrm{TiO}_{2}$ e (b) $\mathrm{TiO}_{2} / \mathrm{WO}_{3}$.

Os resultados dos testes colorimétricos realizado no padrão $\mathrm{P}_{25}$ e nas nanofibras de $\mathrm{TiO}_{2} \mathrm{e}$ de $\mathrm{TiO}_{2} / \mathrm{WO}_{3}$ são apresentados na Tabela I. Os registros para cada amostra foram obtidos com base no sistema CIE- $\mathrm{L} * \mathrm{a} * \mathrm{~b}^{*}$ e, a faixa de medição abrangeu todo o espectro visível (400 a $700 \mathrm{~nm}$ ). A Tabela I também apresenta os valores de luminescência $(\% \mathrm{~L})$, ou seja, a quantidade de luz que é percebida em determinada cor. Se a luminescência $(\% \mathrm{~L})$ for próxima de o\% representa a ausência total de luz refletida (preto) e se for próxima de Ioo\% representa a reflexão total da luz (branco) [6]. $E$ os valores de $\Delta L^{*}$, que informam a respeito das diferenças entre as tonalidades em mais claro ou escuro. Os valores positivos (+) de $\Delta \mathrm{L}^{*}$ indicam a cor mais clara e os valores negativos $(-)$ de $\Delta \mathrm{L}^{*}$ indicam a cor mais escura.

Assim, durante os testes colorimétricos o padrão $\mathrm{P}_{25}$ e as nanofibras de $\mathrm{TiO}_{2}$ tiveram máxima absorbância na região da cor azul-escuro, influência dos valores positivos de a* (cor vermelho) e negativos de $b^{*}$ (cor azul). A tonalidade escura das amostras foi determinada com base nos valores negativos de $\Delta \mathrm{L}^{*}$. As nanofibras de $\mathrm{TiO}_{2} / \mathrm{WO}_{3}$ tiveram máxima absorbância na região da cor azul-claro, influência dos valores negativos de a* (cor verde) e negativos de b* (cor azul). A 
tonalidade clara das amostras foi determinada com base nos valores positivos de $\Delta \mathrm{L}^{*}$. A máxima absorbância na região da cor azul alcançada pelas nanofibras de $\mathrm{TiO}_{2}$ e de $\mathrm{TiO}_{2} / \mathrm{WO}_{3}$ já era esperada, visto que ambas as soluções precursoras (Figura 4) possuem coloração amarela, diferindo apenas na tonalidade. E, em análises colorimétricas o máximo de absorbância ocorre na região da coloração complementar e, neste caso a cor complementar ao amarelo é o azul.

Tanto o padrão $\mathrm{P}_{25}$ como as nanofibras de $\mathrm{TiO}_{2}$ e de $\mathrm{TiO}_{2} / \mathrm{WO}_{3}$ tiveram uma boa quantidade de luz percebida, conforme os valores de luminescência (\%L) demonstrados na Tabela I.

Tabela I. Resultados colorimétricos das amostras.

\begin{tabular}{|c|c|c|c|c|c|}
\hline Amostras & $a^{*}$ & $b^{*}$ & $\begin{array}{c}\text { Diferença de tonalidade } \\
\text { (claro/escuro) } \\
\Delta \mathrm{L}^{*}\end{array}$ & Cor absorvida & $\% \mathrm{~L}$ \\
\hline Nanofibras $\mathrm{TiO}_{2} 650^{\circ} \mathrm{C}$ & $+\mathrm{I}, 47$ & $-12,51$ & $-35,99$ & Azul-escuro & 83,33 \\
\hline Nanofibras $\mathrm{TiO}_{2} 700^{\circ} \mathrm{C}$ & $+3,29$ & $-2,68$ & $-57,43$ & Azul-escuro & 61,42 \\
\hline Nanofibras $\mathrm{TiO}_{2} 750^{\circ} \mathrm{C}$ & $+7,16$ & $-10,44$ & $-24,32$ & Azul-escuro & 92,14 \\
\hline Nanofibras $\mathrm{TiO}_{2} 800{ }^{\circ} \mathrm{C}$ & $+4,14$ & $-8,21$ & $-42,86$ & Azul-escuro & 86,76 \\
\hline $\begin{array}{c}\text { Nanofibras } \mathrm{TiO}_{2} / \mathrm{WO}_{3} 650 \\
\text { }{ }^{-} \mathrm{C}\end{array}$ & $-3,13$ & $-1,45$ & $+66,91$ & Azul-claro & 59,73 \\
\hline $\begin{array}{c}\text { Nanofibras } \mathrm{TiO}_{2} / \mathrm{WO}_{3} 700 \\
\text { }{ }_{\mathrm{C}}\end{array}$ & $-4,42$ & $-10,37$ & $+58,91$ & Azul-claro & 96,26 \\
\hline $\begin{array}{c}\text { Nanofibras } \\
\underset{{ }_{0} \mathrm{CiO}}{ } \mathrm{TiO}_{2} / \mathrm{WO}_{3} 750\end{array}$ & $-3,48$ & $-11,46$ & $+75,4 \mathrm{I}$ & Azul-claro & 84,69 \\
\hline $\begin{array}{c}\text { Nanofibras } \mathrm{TiO}_{2} / \mathrm{WO}_{3} 800 \\
\text { }{ }^{\circ} \mathrm{C}\end{array}$ & $-5,18$ & $-5,99$ & $+56,68$ & Azul-claro & 85,47 \\
\hline Padrão $\mathrm{TiO}_{2}-\mathrm{P}_{25}$ & $+6,45$ & $-2,23$ & $-32,21$ & Azul-escuro & 83,13 \\
\hline
\end{tabular}

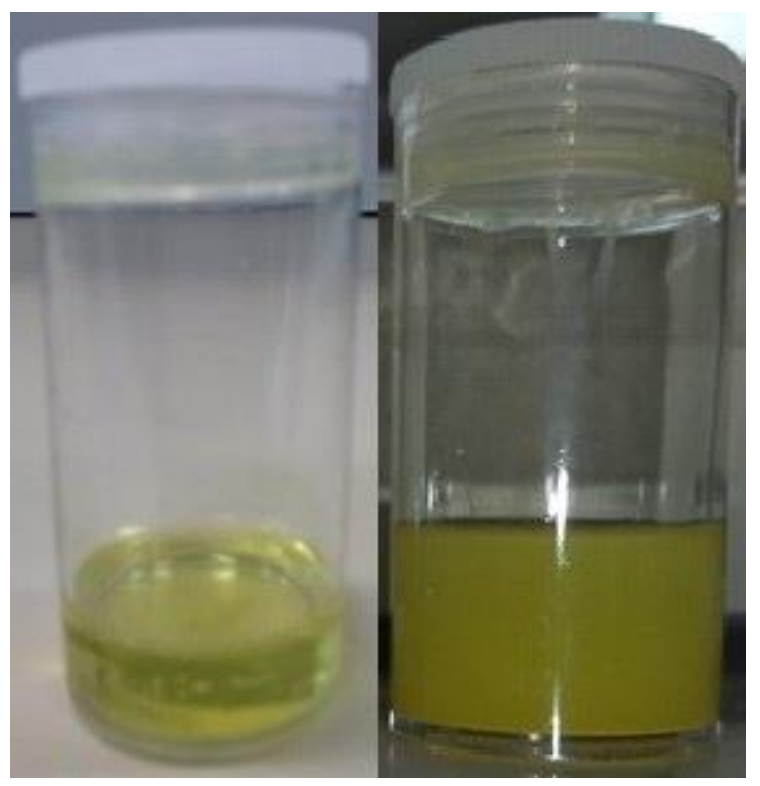

Figura 4. Fotografia da coloração das soluções percursoras antes da síntese das nanofibras por electrospinning. 
A capacidade dos olhos humanos para diferenciar as alterações de cor nas nanofibras foi determinada através dos valores obtidos pela associação da diferença de cor $(\Delta \mathrm{E})$ e a Norma $\mathrm{DIN}$ 6174, Tabela II [13].

Tabela II. Norma DIN 6r74.

\begin{tabular}{|c|c|}
\hline $\begin{array}{c}\text { Diferenças } \\
\left(\Delta \mathrm{E}^{*} \mathrm{ab}\right)\end{array}$ & Classificação \\
\hline $0,0-0,2$ & Imperceptível \\
\hline $0,2-0,5$ & Muito pequena \\
\hline $0,5-\mathrm{I}, 5$ & Pequena \\
\hline $\mathrm{I}, 5-3, \mathrm{O}$ & Distinguível \\
\hline $3,0-6, \mathrm{o}$ & $\begin{array}{c}\text { Facilmente } \\
\text { distinguível }\end{array}$ \\
\hline Maior que 6,o & Muito grande \\
\hline
\end{tabular}

A diferença de cor $(\Delta E)$ mede a capacidade dos olhos humanos para diferenciar as alterações de cor entre os materiais. Esta informação visual apresentou-se distinguível para as nanofibras de $\mathrm{TiO}_{2}$ e facilmente distinguível para as nanofibras de $\mathrm{TiO}_{2} / \mathrm{WO}_{3}$. Estes resultados se basearam nos valores da diferença de cor $(\Delta \mathrm{E})$ apresentados na Tabela III.

Tabela III. Capacidade de percepção visual da cor pelos olhos humanos nas nanofibras sintetizadas.

\begin{tabular}{|c|c|c|}
\hline \multirow{2}{*}{ Amostras } & \multicolumn{2}{|c|}{ Percepção visual da cor } \\
\hline & Diferença de cor $\Delta \mathrm{E}^{*} \mathrm{ab}$ & Classificação \\
\hline Nanofibras $\mathrm{TiO}_{2} 650^{\circ} \mathrm{C}$ & 2,32 & Distinguível \\
\hline Nanofibras $\mathrm{TiO}_{2} 700^{\circ} \mathrm{C}$ & 2,43 & Distinguível \\
\hline Nanofibras $\mathrm{TiO}_{2} 750^{\circ} \mathrm{C}$ & 2,89 & Distinguível \\
\hline Nanofibras $\mathrm{TiO}_{2} 800^{\circ} \mathrm{C}$ & 2,66 & Distinguível \\
\hline
\end{tabular}

Revista Ibero-Americana de Humanidades, Ciências e Educação. São Paulo, v.7.n.1, jan. 2021. 
Revista Ibero- Americana de Humanidades, Ciências e Educação- REASE

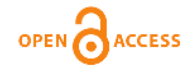

\begin{tabular}{|c|c|c|}
\hline $\begin{array}{c}\text { Nanofibras } \mathrm{TiO}_{2} / \mathrm{WO}_{3} \\
650 \stackrel{\circ}{\circ} \mathrm{C}\end{array}$ & 3,79 & $\begin{array}{l}\text { Facilmente } \\
\text { distinguível }\end{array}$ \\
\hline $\begin{array}{c}\text { Nanofibras } \mathrm{TiO}_{2} / \mathrm{WO}_{3} \\
700 \stackrel{\circ}{\mathrm{C}}\end{array}$ & 3,22 & $\begin{array}{l}\text { Facilmente } \\
\text { distinguível }\end{array}$ \\
\hline $\begin{array}{c}\text { Nanofibras } \mathrm{TiO}_{2} / \mathrm{WO}_{3} \\
750^{\circ} \mathrm{C}\end{array}$ & 3,51 & $\begin{array}{l}\text { Facilmente } \\
\text { distinguível }\end{array}$ \\
\hline $\begin{array}{c}\text { Nanofibras } \mathrm{TiO}_{2} / \mathrm{WO}_{3} \\
800 \stackrel{\circ}{\mathrm{C}}\end{array}$ & 3,59 & $\begin{array}{l}\text { Facilmente } \\
\text { distinguível }\end{array}$ \\
\hline Padrão P25 & $\mathrm{I}, 98$ & Distinguível \\
\hline \multicolumn{3}{|c|}{$\begin{array}{c}\text { Os olhos humanos não conseguem distinguir diferenças de cor }(\Delta \mathrm{E}) \\
\text { menores que I. }\end{array}$} \\
\hline
\end{tabular}

\section{CONCLUSÕES}

Os resultados obtidos com as nanofibras sintetizadas quando da sua utilização em colorimetria e fotocatálise, é devido a sincronicidade existente entre as propriedades químicas e físicas dos óxidos de titânio e tungstênio, e pela ocorrência de fenômenos semelhantes. Em ambos os eventos a luz irradiada, a energia de band gap, o comprimento de onda, e a formação de defeitos (Vacâncias de $\mathrm{O}_{2}$ ) foram essenciais tanto para a percepção visual das diferentes cores quanto para a fotoatividade na degradação do corante alaranjado de metila. Todas as nanofibras puderam ser utilizadas como fotocatalisadores e nos testes colorimétricos. Mesmo as nanofibras de $\mathrm{TiO}_{2}$ tratadas em temperaturas mais elevadas $\left(750^{\circ} \mathrm{C}\right.$ e $\left.800{ }^{\circ} \mathrm{C}\right)$ que tiveram baixa fotoatividade, apresentaram capacidade de degradar o corante alaranjado de metila e absorver cor. A presença de tungstênio aumentou a eficiência fotocatalítica dos materiais, inibiu da recombinação do par elétron/lacuna $\left[(e) /\left(h^{+}\right)\right]$, permitindo a transferência de cargas entre o $\mathrm{TiO}_{2}$ e o $\mathrm{WO}_{3}$. A elevação da temperatura de tratamento térmico possibilitou que as vacâncias de $\mathrm{O}_{2}$ adquirissem a mobilidade necessária para passar para um estado desordenado na rede, aumentando a capacidade de degradação e absorção de luz.

\section{AGRADECIMENTOS}

Os autores agradecem ao apoio financeiro da Universidade Federal do Rio Grande do Sul (UFRGS), da Coordenação de Aperfeiçoamento de Pessoal de Nível Superior (CAPES) e do CNPq. 


\section{REFERÊNCIAS}

[I] W. D. Jr. Callister, Ciência e Engenharia de Materiais: Uma introdução, $5^{\underline{a}}$ edição, LTC, Rio de Janeiro (2002) p.589.

[2] L. C. Lopes, Controle metrológico da cor aplicado à estamparia digital de materiais têxteis. 2009. Iozp. Dissertação de Mestrado (Mestrado em Metrologia para a Qualidade e Inovação) -Programa de Pós-Graduação em Metrologia, Pontifícia Universidade Católica do Rio de Janeiro.

[3] C. G. O. Griebeler, Colorimetria da madeira de eucalyptus grandis w.hill ex maiden modificada termicamente, 2013, 69p. Dissertação de Mestrado (Tecnologia e Utilização de Produtos Florestais) - Programa de Pós-Graduação em Engenharia Florestal, Universidade Federal do Paraná, Curitiba. [4] Y. M. Chiang, D. Birnie, W. D. Kingery, Physical Ceramics Principles for Ceramics Science and Engineering, ${ }^{\mathrm{a}}$ edição, Wiley, New York (1997) p.544.

[5] H. Zollinger, Color Chemistry Synthesis, properties, and applications of organic dyes and pigments, $2^{2}$ edition, Wiley, Universidade de Michigan (1991) p.496.

[6] K. Minolta, Precise color communication: color control from feeling to instrumentation, Osaka, Japan (2007) p.62.

[7] P. C. M. Tiano, Cor e colorimetria: minicursos CRQ-IV, estado de São Paulo, (2009).

[8] J. FElTRIN, Jr. SARTOR, M. N. A. DE NONI; A. M. BERNARDin, D. HOTZA, J. A. LABRINCHA, Superfícies fotocatalíticas de titânia em substratos cerâmicos. Parte I: Síntese, estrutura e fotoatividade, Cerâmica, 2013, v.59, pp.62o-632.

[9] N. Rahimi, R. A. Pax, E. G. Mac, Review of functional titanium oxides. I: $\mathrm{TiO}_{2}$ and its modifications, Progress in Solid State Chemistry, 2016, v.44, pp. 86-105.

[ı] E. N. S. Muccillo, Condutores de íons oxigênio - uma breve revisão, Cerâmica, 2008, v.54, pp.i29I44.

[II] B. Bharti, S. Kumar, H. N. Lee, K. Rajesh, Formation of oxygen vacancies and $\mathrm{Ti}^{3+}$ state in $\mathrm{TiO}_{2}$ thin film and enhanced optical properties by air plasma treatment, Scientific Reports, 2016, pp.I-I2.

[12] H. G. OLIVEIRA, Eletrodos porosos contendo $\mathrm{TiO}_{2}$ e $\mathrm{WO}_{3}$ : propriedades eletroquímicas e atividade fotocatalítica para remoção do corante rodamina $6 \mathrm{G}$ e do hormônio i7 $\alpha$-etinilestradiol em solução aquosa. 2012. I88p. Doutorado. (Tese de doutorado em Ciências) Instituto de Química da Universidade Estadual de Campinas SP/Campinas.

[13] R. A. Silva, C. O. Petter, I. A. H. Schneider, Color loss evaluation of artificially stained agates. REM: Revista Escola de Minas, 2007, v.6o, pp.477-482. 